\title{
Larking Around or Malicious Malarkey? The Impact and Management of workplace Banter
}

\author{
Arwen Wilcock, Phil James and Kate Cooper
}

\begin{abstract}
Banter is intrinsic human behavior, it is multifunctional, facilitating social interaction, building rapport and tackling complex and challenging topics of conversation [1]. In light of prevalent social media campaigns challenging acceptance of banter and associating it with poor conduct, such as bullying and harassment, we aimed to understand its role and impacts within workplaces.

Surveying over 1000 people, the most commonly accepted topics of banter were age, gender and personality, while unacceptable topics included physical and mental health, religion/faith, sexual orientation and ethnicity). We found that although the majority felt banter had a positive role, and would not ban it, inappropriate banter was significantly damaging to workers mental health and negatively impacted confidence. Furthermore, we found that women were more negatively affected than men, as were younger and less experienced employees. A significant proportion of respondents (around half) reported challenging inappropriate banter; however, women and younger/less experienced staff as well as newly promoted managers felt less enabled to tackle inappropriate banter.

With one in five women losing confidence, one in ten women experiencing mental health problems and 1 in 25 people leaving their jobs due to inappropriate banter (according to our findings), banter is potentially damaging to employee's wellbeing and organisational productivity. Our research makes a strong case for organisations to review and update policies and procedures relating to banter, bullying and harassment and for organisations to provide training to identify and address inappropriate banter.
\end{abstract}

Keywords - Management, Leadership, Banter, Bullying, Harassment, Inequality.

\section{INTRODUCTION}

Banter "the playful and friendly exchange of teasing remarks" [2] is an integral part of human behaviour. Forming a normal part of human social development, it evolves from unwanted teasing in younger children into a more sophisticated tool to share experiences, engage in difficult topics and build relationships [1]. The role of banter as a social device in the workplace has come under scrutiny in the public eye, particularly in light of the \#metoo campaign, which has garnered international momentum as a stand against sexual harassment and assault [3]. However, despite the prevalence of and commentary about banter, research into its role within the

Arwen Wilcock, Research Manager, The Institute of Leadership and Management Pacific House, Relay Point, Tamworth, B77 5PA. UK

Phil James and Kate Cooper, The Institute of Leadership and Management Pacific House, Relay Point, Tamworth, B77 5PA. UK. workplace is sparse and limited mainly to a handful of academic studies and white papers examining wider issues of bullying, harassment and inequality.

While we have established, above, that banter is part of a normal social activity, within the restaurant sector banter is not only an inescapable accepted 'norm', but is also seen as a tool to initiate and socialise new staff [4]. The "survival" of new staff, who are exposed to significant amounts of banter, is frequently viewed by chefs and restaurant staff as a rite of passage and as a way of gauging an individual's "fit" (or not) for that particular workplace [4]. Unfortunately, in this case, the topics and application of banter crossed the line into bullying and harassment, and the most vulnerable staff, female staff and staff with identifiable differences frequently left due to the harassment.

A key challenge for individuals participating in banter seems to be identifying when banter is acceptable, fun and used in a way to foster inclusivity as opposed to being discriminatory, unwanted, threatening or oppressive. At the level of legislation, it is also challenging to define acceptable conduct for banter. Within the UK, under article 8 of the 1998 Human Rights Act, workers have the right to a private and family life, however banter may breach these basic human rights [5]. Additionally, article 14 state that workers have the right to freedom of religion, freedom of expression and freedom from discrimination. While certain forms of banter could easily breach Human Rights legislation, it is note worthy that no legal protection from banter exists in law; the only access to legal protection for conduct relating to banter is via bullying and harassment legislation where banter is inferred not specifically stated. There are suggestions that new legislation should be enacted to provide clarity and definition that identifies when workplace banter has become unacceptable [5].

Although current legislation is vague, there are mounting numbers of reports in news and social media outlets that there are some serious and significant issues with how banter is used across all sectors. Additionally, the TUC (a UK trade union) reported that the instigator of banter frequently views their action as 'harmless fun', and when there is a problem they even go so far as to blame the subject of the banter for being unable to take a joke [6]. In contrast, the subject of the banter reported frequently feeling undermined, humiliated and at times terrified from experiencing certain forms of banter. In 2016 one union found $32 \%$ of women had been the subject of 
unwelcome banter in the workplace that had a sexual overtone. Furthermore, only 1 in 5 women were reporting sexual harassment in the form of banter, indicating there is fear about being taken seriously and having appropriate follow up [6]. As a result of its research the union called for all organisations to change their workplace practices to protect individuals from being victims of banter used for the purpose of harassment [6].

With an understanding that banter evolves as a behaviour that is a normal part of social development and relationship building [1], but with recognition that banter as a behaviour can easily become bullying and harassment, we investigated its use and role within the workplace. We found that predominantly banter was viewed as having a positive role in building relationships and learning to get along with team members. However, our data revealed concerning statistics indicating that when banter is abused its impact can lead to individuals avoiding their colleagues and in more serious cases mental health problems and leaving the place of employment.

\section{RESULTS}

To undertake this research, we surveyed 1025 members of the Institute of Leadership and Management, data was analysed by the whole population and demographic subpopulations (gender, age, type of role).

\section{A. Use of banter in the Workplace}

The majority of respondents, 98\%, reported that they experience some form of workplace banter. Despite evidence that banter is used for bullying and harassment (outlined above), $73 \%$ stated that they would not ban it (Figure 1a); indicating that the majority view banter as an acceptable and integral element of work culture. We asked respondents to identify topics of banter that they participate in and interpreted this as topics respondents view as acceptable for banter (Figure 1b). We also asked respondents to identify topics of banter that they hear in the workplace and topics that they believe are unacceptable (Figure 1c and d). The most taboo topics of banter that respondents said were unacceptable within the workplace included banter about medical/health $(68 \%)$, Sexual orientation $(65 \%)$, religion/faith $(62 \%)$ and ethnicity $(62 \%)$, the most common topic of banter respondents experiences was age $(71 \%)$.

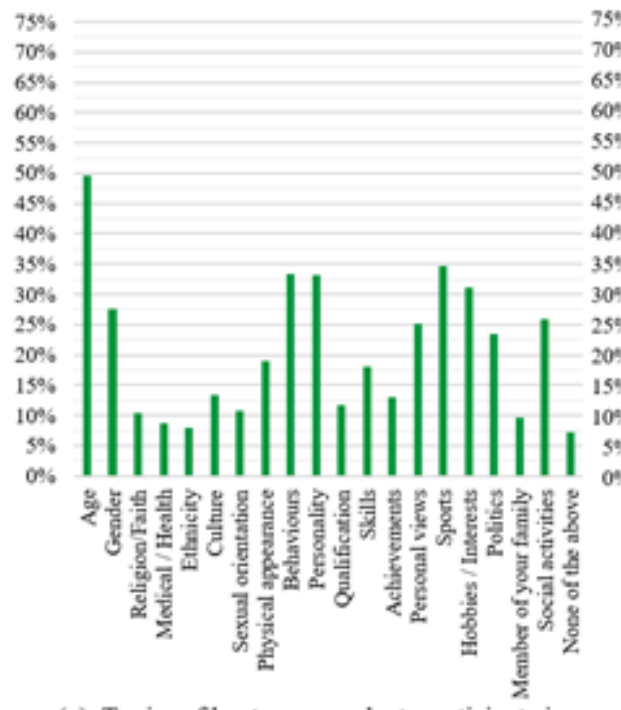

(a). Topics of banter respondents participate in

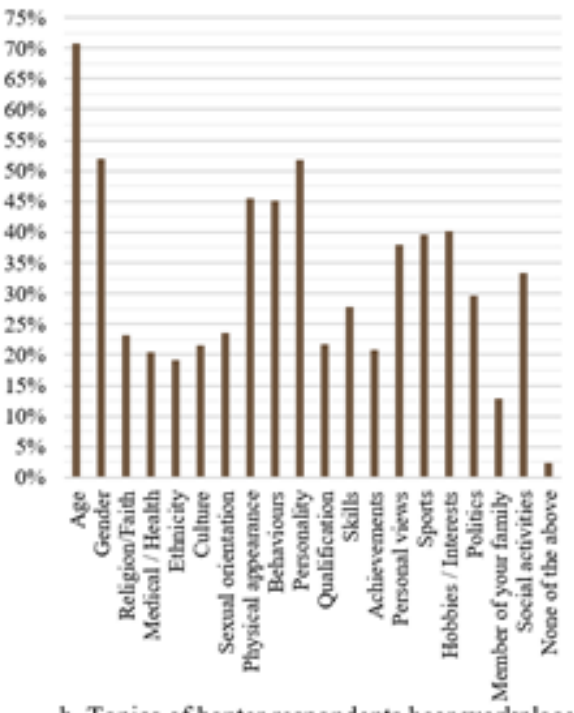

b. Topics of banter respondents hear workplaces

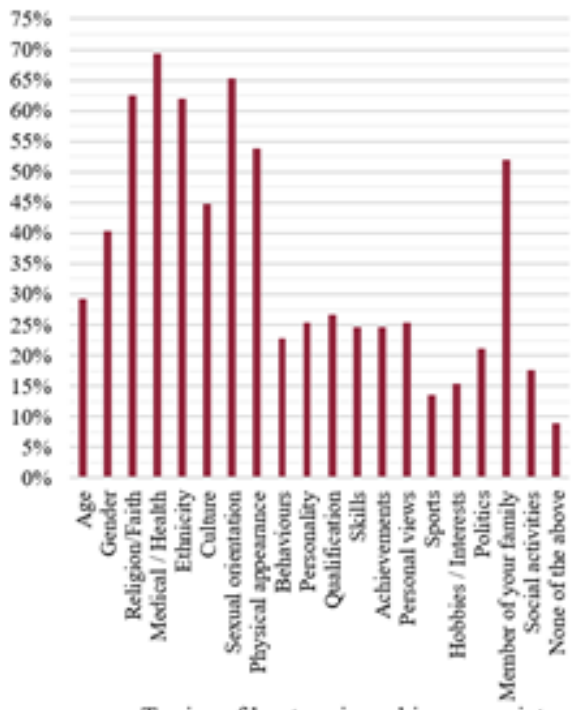

c. Topics of banter viewed inappropriate

Fig. 1: Topics of banter in the workplace; percent of people reporting (a) topics participated in by respondents, (b) topics heard by respondents, (c) topics viewed inappropriate for workplaces.

We also asked participants if their organisations had formal policies about banter, $26 \%$ of respondents said yes, while $37 \%$ said no and the other $37 \%$ were unsure (this response did not differ much between demographic groups) (Figure 2). This suggests that the subject of banter is likely to be neglected in many workplace policies and procedures.

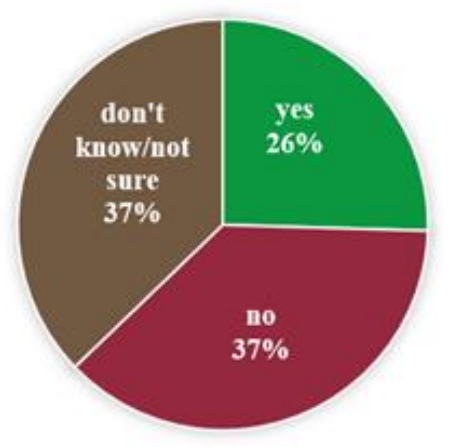

Fig. 2: Does your workplace have a policy on banter? 


\section{B. Negative Impacts of Banter; Women are More Impacted than Men}

We next wanted to understand the effect banter has on workers and understand if respondents associated workplace banter with negative personal, social, or mental health impacts. We found $10 \%$ of respondents reported avoiding workplace situations due to banter; it is worth noting that if 1 in 10 of the workforce avoids workplace situations there is likely to be a significant impact on productivity, especially within a collaborative work environment. While we found little difference in the proportion of men and women feeling embarrassed by banter ( $18 \%$ of men and $20 \%$ of women), we found that it had an overall larger negative impact on women. Mental health was negatively impacted in $10 \%$ of women, compared to $3 \%$ of men. Confidence was impacted, $20 \%$ of women reporting loss of confidence in contrast $9 \%$ of men reported it impacted their confidence negatively.

When we examined the differences between role types, we found that graduate trainees were most likely to be demotivated by inappropriate banter, $21 \%$ compared to the average of $10 \%$. However, only $3 \%$ of graduate trainees were likely to avoid work socials compared to $26 \%$ of people in their first employed role. When it comes to loss of confidence people in their first role and first-time managers are most affected, with $21 \%$ and $19 \%$ respectively stating they lost confidence, compared to $12 \%$ of graduate trainees. First time mangers reported the greatest impact on their performance with $11 \%$ stating their performance was affected negatively. When we looked at the impact of banter in different age groups we consistently found that the 31-40 age group were more affected than any other age group with loss of confidence $(19.5 \%)$, drop in performance $(10.5 \%)$, mental health $(10 \%)$, avoiding work situations (16\%), avoiding work socials (13\%). Our data demonstrates that a significant proportion of the workforce, ranging from 1 in 10 to 1 in 4 people are negatively affected by inappropriate banter.

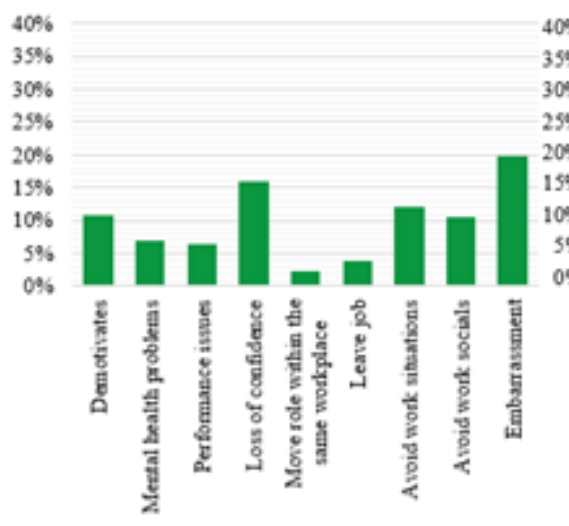

(a) Whole population

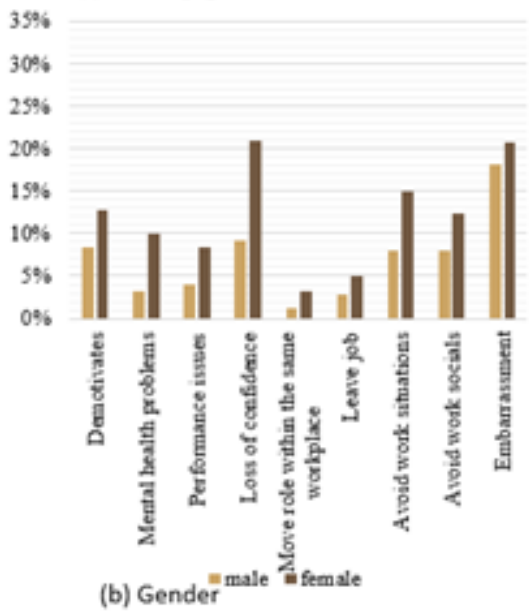

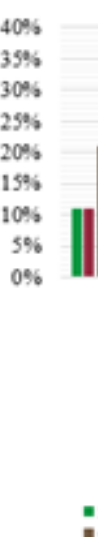

afint job

Firat time trainee

Senicr manapement team

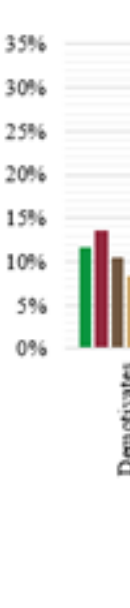

Fig. 3: Negative impacts of banter on the workforce by different demographic groups; a. the whole population, b. male and female staff, c. different role types, d. different age groups

\section{C.Perspectives of workers on banter and it's use within the workplace}

Many people in our research were supportive of banter when they feel it is used appropriately and not used for bullying or harassment; over $75 \%$ saying they would not ban banter from the workplace, about 5\% said they would ban banter, the rest were unsure (Figure 4). 


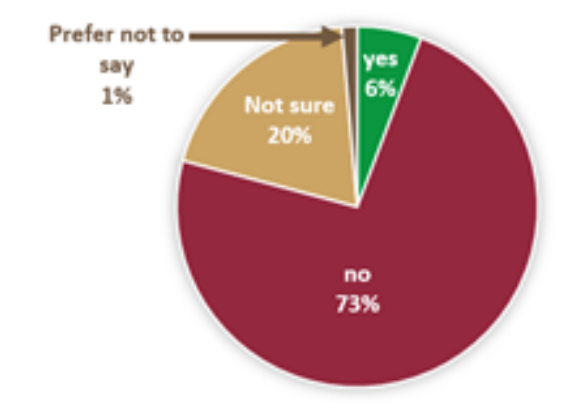

Fig. 4: Should Banter be Banned?

We asked participants if, and when, banter might be used appropriately within the workplace, many positive reasons were given. We found men were slightly more positive about banter than women, but little difference in the other demographic subgroups.

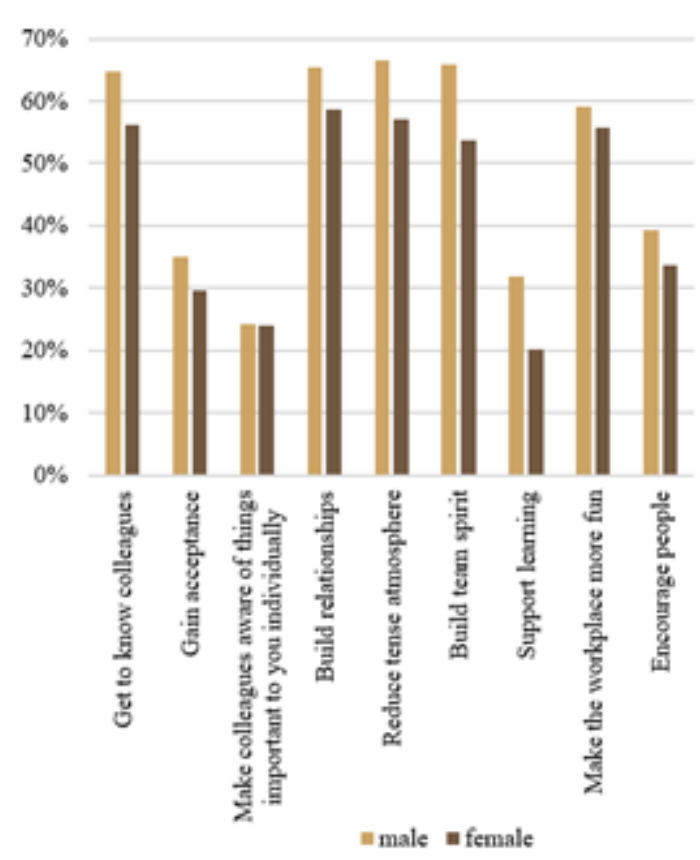

(a) Positive reasons for using banter

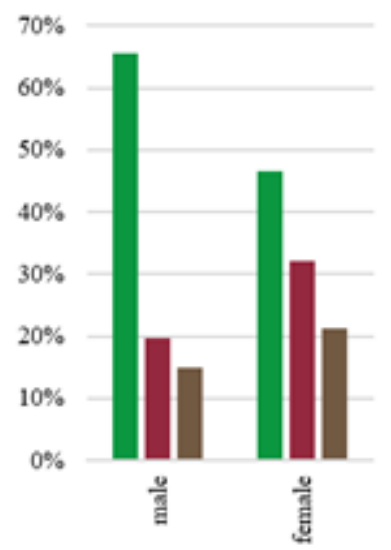

ayes

No

= Don't know/not sure

(b) Do you initiate banter where you are the subject?

Fig. 5: Reasons for using banter (a) Positive reasons for using banter, (b) Do respondents make themselves the subject of banter?

\section{Older people, more senior staff and men are more likely to challenge banter}

We wanted to understand how people respond within the workplace when confronted with banter that they feel inappropriate (Figure 6).

Not surprisingly, we found that people who were older (70\%) or who had more senior positions (79\% of senior managers and $71 \%$ of experienced managers) were more likely to challenge or report inappropriate banter than their younger (46\%) or less senior colleagues $38 \%$ in their first job and $40 \%$ of graduate trainees). We did, however find, the responses between the genders quite interesting. Of those who had experienced inappropriate banter, men were more likely than women to directly challenge the behaviour, $73.5 \%$ compared to $55.3 \%$ respectively. In contrast women were more likely than men to seek other forms of support to address issues about banter, for example; women are more likely to confide in family/partner $(22 \%$ compared to $8.4 \%)$, work colleague/friend ( $22 \%$ compared to $14 \%$ ), confide in a friend outside work (14.4\% compared to $7 \%$ ) or report it to a manger (20\% compared to $16 \%)$. 


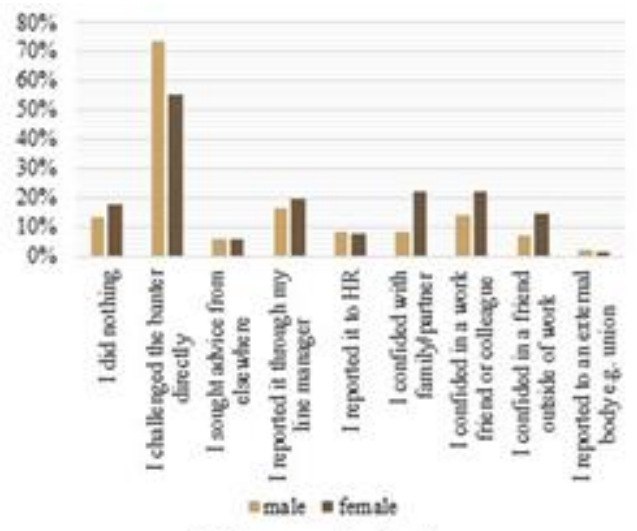

(a) Responses by Gender

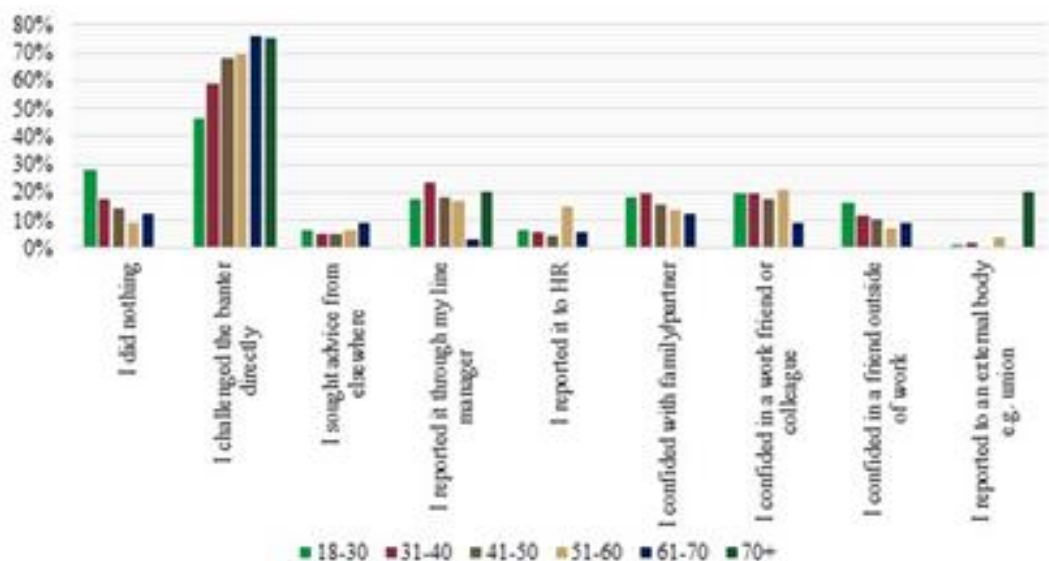

(b) Responses by Age

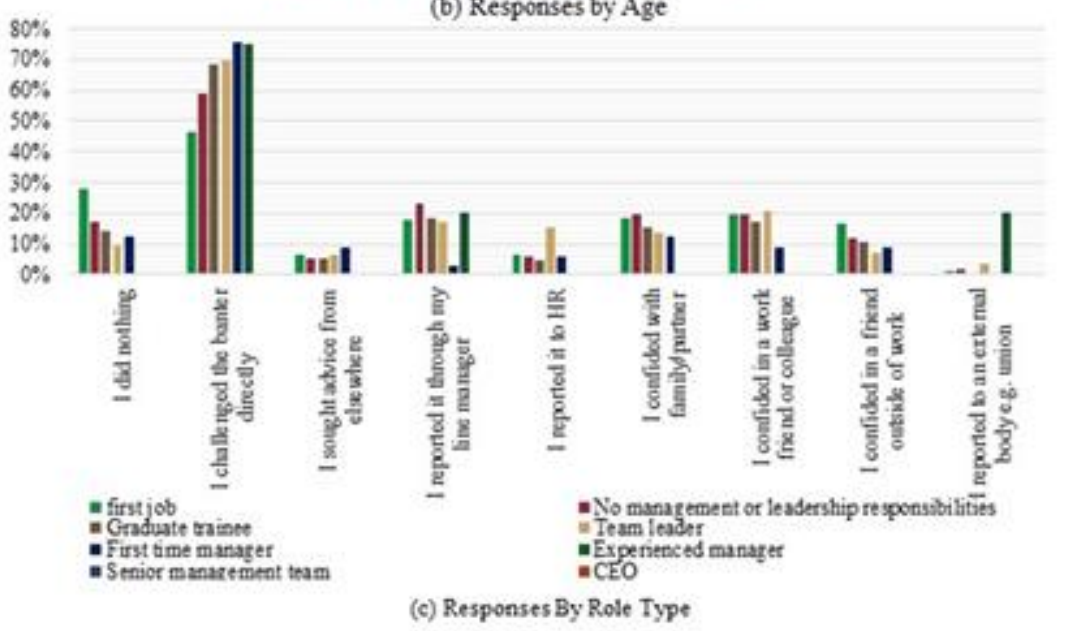

Fig 6. How people in different demographic groups respond to workplace banter (a) by gender, (b) by age, (c) by role type.

We asked respondents to tell us about some of the actions they had taken, while some reported only needing to speak to the initiator to resolve issues, some reported more serious interventions, such as following performance management and suspension routes; one responded, "I've been in the position where I've suspended someone". While the majority reinforced data shown in Figure 6, we had some responses that were significantly more alarming. The most extreme experience reported was one of sexual harassment that was subsequently endorsed by the line manager; "I asked my line manager for help. The person had taken the jokes about my appearance, gender etc too far. My line manager said that it was a personal matter between us, that I should fix it by "having sex" with the man involved as he was flirting with me. The guy made my work life hell and I considered leaving the company and industry over it". While this is an extreme case, it is very concerning that this type of behaviour not only takes place but is endorsed by managers in positions of power; our data shows that inappropriate banter has resulted in a $4 \%$ people (just under 1 in 25) leaving their organisation, and $2 \%$ moving role within their organisation.

\section{CONCLUSIONS}

Overall, we found that banter is a widely accepted and expected behaviour within the work place. The majority favour banter as a way to engage socially with colleagues to foster and develop professional relationships and to make work a more enjoyable experience (Figure 5). Indeed, the majority view it positively with $73 \%$ saying that workplace banter should not be banned (Figure 4).

We found that more women are negatively affected by banter than men, in most issues we examined the proportion of women affected was around double that of men. Although our research does not determine why this is, it suggests that women and minority social groups frequently experience banter as a form of bullying and harassment $[4,6]$. We also found that significant more women, and younger employees reported not tackling banter when they felt it was inappropriate. Our findings are supported by research from the TUC, stating many women are unlikely to challenge inappropriate workplaces behaviours due to fear of repercussions [6].

A small but significant proportion of the population is affected negatively by inappropriate banter, ranging from 1 in 10 to 1 in 4 people, leading to demotivation, mental ill health, avoiding spending time with colleagues and in the extreme 1 in 25 leaving their job. This numbers of people affected would indicate that there is likely to be negative implications for productivity and overall organisation performance. There is, therefore, clearly a need for organisations to address and 
tackle poor behaviours. Although the majority of respondents stated they would not ban banter, we found that only one third were confident that their organisations had appropriate policies and procedures in place. Furthermore, banter may be used within the workplace in ways that breach basic human rights [5]. Although our respondents provide very balanced views of banter, for example one stated "I feel that it has been part of life from the playground to work place, it is part of our culture and I have experienced this across the different countries that I have worked", it is important to have easily accessible support available for anyone that is a victim of unwanted behaviour. This would include organisations undertaking comprehensive review of bullying and harassment policies, with emphasis given to minority groups and protected characteristics. Clear policies and procedures that identify how to raise concerns, should be supported with tailored training and development that gives emphasis to emotional intelligence and cultural awareness. Alongside this, organisations need to foster a supportive and empowering culture where they feel able to raise concerns if they feel inappropriate banter takes place. Finally, organisations should invest in understanding how behaviours and the potential impact of banter influences gender inequality.

\section{METHODOLOGY}

\section{A. Data and Sample}

The Institute of Leadership \& Management undertook an on-line survey in 2018 , with $n=1025$ respondents completing the survey. The data was analysed at the whole population level and by demographic groups as follows; gender, male $\mathrm{n}=443$, female $\mathrm{n}=567$; age, $28-30 \mathrm{n}=162,31-40 \mathrm{n}=266,41-50$ $\mathrm{n}=303,51-60 \mathrm{n}=240,61-70 \mathrm{n}=41,70+\mathrm{n}=5$ (note that due to the low numbers over 70 data is not discussed in the text, but data is provided for interest); no management or leadership responsibilities $n=158$, first job $n=19$, graduate trainee $n=33$, team leader $n=156$, first time manager $n=150$, experienced manager $n=330$, senior management team $n=156, C E O=23$.

\section{B. Measures}

Respondents were asked a number of questions about the use of banter within the workplace, this included; identifying acceptable and unacceptable topics, positive and negative uses of banter, the impact of banter on them within the workplace both positive and negative, how they responded to inappropriate banter and whether their workplace had official policies about banter. The fields of data collection are shown within the figures.

Most of the questions were closed questions asking respondents to identify statements that reflected their experiences of, or responses to workplace banter. Respondents were also given the opportunity to provide free text responses reflecting about their own experiences of work place banter (positive, negative or neutral). The survey was conducted in line with the Market Research Society (MRS) Code of Conduct. All responses were anonymous, but respondents were asked if they would be willing to be contacted for PR purposes and were also incentivised to participate in the survey.

\section{REFERENCES}

[1] C. B. Mills, "Child's play or risky business? The development of teasing functions and relational implications in school-aged children," Journal of Social \& Personal Relationships, vol. 35, pp. 287-306, 2018. https://doi.org/10.1177/0265407516683557

[2] OED. 2018. "banter - Definition of banter in English", Oxford $\begin{array}{llll}\text { Dictionaries. } & \text { Accessed } & 1 & \text { May }\end{array}$ https://en.oxforddictionaries.com/definition/banter

[3] Sini, R., (2017) "How 'MeToo' is exposing the scale of sexual abuse", BBC News. London. www.bbc.co.uk/news/blogs-trending-41633857

[4] C. Giousmpasoglou, E. Marinakou, and J. Cooper, "'Banter, bollockings \& beatings': the occupational socialisation process in Michelin-starred kitchen brigades in Great Britain and Ireland," International Journal of Contemporary Hospitality Management, vol. 8 pp. 1882-1902, 2018 . https://doi.org/10.1108/IJCHM-01-2017-0030

[5] S. Middlemiss, “'Another nice mess you've gotten me into' employers' liability for workplace banter" International Journal of Law and Management, vol. 59, pp. 916-938, 2017 https://doi.org/10.1108/IJLMA-07-2016-0063

[6] TUC. "Still just a bit of banter?" Trades Union Congress. London. 2016.. 